\title{
Caracterización morfológica de caninos en premolares, un caso de anodoncia parcial de caninos maxilares.
}

\author{
Morphological characterization of canine teeth done in first \\ bicuspid, a maxillary canine partial anodontia case report.
}

Pablo César Martínez Cardona,* José Martínez Zepeda,* Francisco Espinosa,* Yuridia Michelle Ávalos Equihua*

\section{RESUMEN}

En este informe de caso clínico se muestra el tratamiento exitoso de un paciente con anodoncia parcial de órganos dentales 13 y 23 debido a que fueron extraídos por presentar impactación y mal pronóstico de tracción. En este artículo se presenta un paciente masculino de 29 años clase I esquelética con un perfil convexo, maloclusión de clase III, overjet disminuido, ausencia de guías funcionales, discrepancia oseodentaria positiva en arcada superior y negativa en arcada inferior. El tratamiento se realizó con extracciones de los órganos dentales 34 y 44 para nivelar las discrepancias óseo dentarias interarcadas, se llevó a cabo mediante un cierre de espacios recíproco por medio de cadenas elásticas para ambas arcadas, con lo que se logró crear un overjet y overbite adecuados. La creación de las guías caninas funcionales se consiguió mediante el cambio de morfología de los órganos dentales 14 y 24, los cuales fueron llevados a la posición de los caninos ausentes. El tiempo total de tratamiento para este paciente fue de 24 meses. Se realizaron ameloplastias positivas, la aplicación de agregados de resina para mejorar la funcionalidad y proveer salud articular. Se sugiere que ante casos de anodoncia de caninos en la arcada superior, un tratamiento favorable se puede llevar a cabo mediante el cierre de espacios, la caracterización morfológica de los caninos ausentes mediante ameloplastias positivas en premolares.

Palabras clave: Anodoncia parcial, caracterización morfológica de caninos, ameloplastias, extracciones.

\section{ABSTRACT}

This case report shows the successful treatment of a patient with partial anodontia of dental organs 13 and 23 because they have been extracted due to present impactation and poor traction prognosis. This article presents a 29-year-old male class I skeletal patient with convex profile, class III malocclusion, overjet reduction, absence of functional guidance, positive bone-teeth discrepancy in the upper arch and negative in the lower arch. The treatment was carried out with the extractions of the dental organs 34 and 44 for correcting oral dental discrepancies between upper and lower arches; it was done using reciprocal closing of spaces by using elastic chains for both arches in order to achieve a suitable overjet and overbite. The creation of the canine guides was achieved by changing the morphology of the dental organs 14 and 24, which were taken to the position of the absent canines. The total treatment timing for this patient was 24 months. Positive ameloplasties were performed by application of resin aggregates to improve functionality and provide joint health. A favorable treatment for these kinds of cases of canine anodontics in the upper arch is carried out by closing spaces and the morphological characterization of the absent canines by positive in-premolar ameloplasties.

Keywords: Partial anodontia, Morphological characterization of canine teeth, ameloplasty, extraction.

\section{INTRODUCCIÓN}

$\mathrm{L}$ os dientes caninos son considerados muy impor$\downarrow$ tantes para el sistema estomatognático, siendo indispensables para los movimientos de lateralidad y vitales para la integridad de los arcos dentarios, responsables para el funcionamiento y la armonía oclusal. Su posición adecuada en la arcada asegura un buen contorno de la cara y una estética adecuada de los dientes. ${ }^{1}$

\footnotetext{
* Maestría en Ortodoncia de la Universidad de La Salle Bajío, León, Guanajuato, México.
}

Recibido: 27 de noviembre de 2018. Aceptado: 19 de julio de 2021.

Citar como: Martínez CPC, Martínez ZJ, Espinosa F, Ávalos EYM. Caracterización morfológica de caninos en premolares, un caso de anodoncia parcial de caninos maxilares. Rev ADM. 2021; 78 (4): 215-220. https://dx.doi.org/10.35366/101076 
La retención de los caninos es una anomalía en la cual el diente se encuentra impedido para erupcionar, ya sea por obstrucción ocasionada por hueso o por dientes adyacentes dentro de otras complicaciones fisiológicas. Puede ser considerada una vez que la erupción se ha demorado y/o existe evidencia clínica o radiográfica de que no tendrá lugar. ${ }^{2}$

Dependiendo de una serie de factores como la posición del diente retenido en relación a los dientes adyacentes, angulación, distancia que el diente debe ser movido, dilaceración radicular y posible presencia de anquilosis o reabsorción radicular, se debe decidir la terapéutica más conveniente como medios o métodos de abordaje para traccionarlo para su inclusión en la arcada; si esto no fuera posible, se deberían plantear otros tratamientos como la extracción o el transplante. ${ }^{3,4}$

La extracción del canino incluido puede afectar a la estética y oclusión, pero a veces es la única opción si hay limitaciones en llevar al canino a su posición en la arcada o su anatomía está afectada. ${ }^{5}$

La ausencia de caninos presenta una condición que complica el tratamiento ortodóncico, y con ello aparece una disyuntiva: hacer el cierre ortodóncico o abrir el espacio para que sea restaurado con un implante $y / 0$ prótesis. Al manejar estos casos, se deben tomar en cuenta aspectos como: tipo de maloclusión, condición de las piezas adyacentes, requerimientos de espacio y edad del paciente. El tratamiento ideal es la opción más conservadora que pueda satisfacer los requerimientos individuales de estética y función. .,7 $^{2}$

\section{PRESENTACIÓN DEL CASO}

Diagnóstico y etiología: hombre de 29 años que acudió a la maestría en Ortodoncia de la Universidad De La Salle Bajío con el motivo de consulta «quiero arreglar los espacios entre mis dientes».

Al inicio, se tomaron registros clínicos para su análisis diagnóstico como fotografías de estudio extraorales e intraorales (Figura 1), modelos montados en articulador Panadent (PANADENT $\left.{ }^{\circledR}, \mathrm{CA}, \mathrm{EUA}\right)$, radiografías, exploración física de articulación temporomandibular y exploración clínica para realizar análisis ortodóncico dental. Una vez realizada la toma de registros se realizaron las siguientes observaciones.

Aspectos estéticos: el paciente presentó un perfil convexo, una asimetría de Williams del lado derecho con una desviación mandibular de $3 \mathrm{~mm}$ hacia la derecha, un biotipo dolicofacial con un tercio inferior, ángulo nasolabial en norma, ángulo mentolabial obtuso, posición sagital del labio superior en norma, labio inferior y mentón levemente retruídos. Sonrisa plana e incompleta sin exposición de corredores bucales. Línea media dental superior coincidente con línea media facial y desviación de línea media dental inferior $3 \mathrm{~mm}$ a la derecha respecto a la media facial, exposición de incisivo central superior en reposo de $1 \mathrm{~mm}$, competencia labial, proyección de labio superior de $5 \mathrm{~mm}$, proyección de labio inferior de $-0.5 \mathrm{~mm}$, proyección de mentón de $-4 \mathrm{~mm}$, taper labial de $2 \mathrm{~mm}$, con competencia labial.

Aspectos esqueléticos: se observaron bases apicales coincidentes, un patrón general mesofacial, clase I esquelética, patrón dolicofacial del tercio inferior (Figura 2), inclinación dolicofacial del cuerpo mandibular con mandíbula mesofacial, pobre crecimiento sagital mandibular moderado, posterorrotación del cuerpo mandibular, una base craneal plana, posición normal moderada del maxilar, crecimiento vertical facial posterior en norma y alto potencial de crecimiento de la rama mandibular.

Aspectos dentoalveolares: se consideró la discrepancia oseodentaria positiva en arcada superior de $7 \mathrm{~mm}$, discrepancia oseodentaria negativa en arcada inferior de $-7.9 \mathrm{~mm}$, proinclinación de incisivos centrales inferiores, proinclinación de incisivos centrales superiores, línea media dental inferior desviada $3 \mathrm{~mm}$ hacia la derecha respecto a línea media dental superior, overbite incisivo $1.5 \mathrm{~mm}$, curva de Spee de $1 \mathrm{~mm}$, relación molar clase III bilateral, relación incisiva clase III, overjet incisivo 0.5 $\mathrm{mm}$ (Figura 3), desgaste dental, ausencia de caninos y también condiciones morfológicas específicas, como la fusión de los órganos dentales 41 y 42 a nivel coronal, así como geminación en órgano dental 27.

Aspectos periodontales y funcionales: se observó un estado periodontal sano, márgenes gingivales no aceptables, punto de contacto prematuro en RC a nivel de 27 y 37 cúspide-cúspide (Figura 4), ausencia de guías funcionales, rangos de máximas excursivas en normalidad (lateralidades y protrusión normales).

\section{Objetivos terapéuticos}

Basados en las características analizadas desde el punto de vista ortodóncico, se trazaron objetivos de tratamiento específicos, aliviar la discrepancia oseodentaria positiva en la arcada superior así como la discrepancia negativa que se determinó en la arcada a inferior, eliminar desgaste dental que se presenta a causa de un trauma oclusal provocado por contactos dentales inadecuados manifestados en una oclusión borde a borde a nivel del segmento anterior, mejorar relación incisiva que se encuentra reducida 

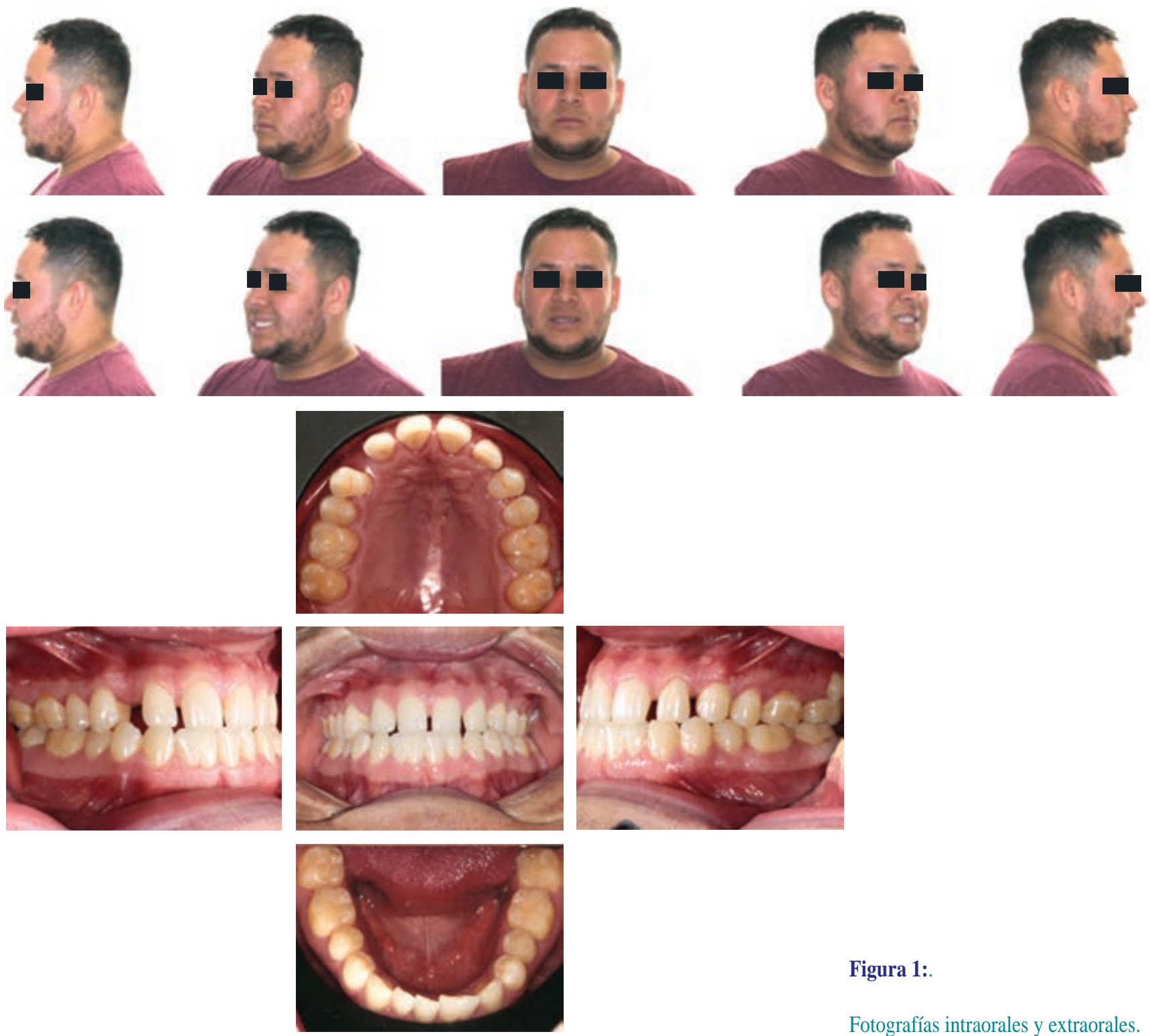

Figura 1:

Fotografías intraorales y extraorales.

por la mala posición dada por adaptación causada por los espacios de anodoncia de los caninos.

Se estableció como uno de los objetivos principales crear una relación canina estética y funcional para dar estabilidad a la salud articular y a la oclusión a largo plazo, así como para evitar contactos funcionales inadecuados causantes de la atrición dental, dicha relación sería creada por medio de la caninización de premolares 14 y 24 mediante ameloplastias negativas y positivas; mejorar relación molar por medio de movimientos dentales para establecer un correcto funcionamiento y estabilidad a niv- el posterior; conseguir sobremordidas horizontal y vertical que complementarán el correcto funcionamiento de las guías funcionales que se planearon durante el tratamiento.

Mejorar proyección de tejidos blandos fue otro de los objetivos, siempre es importante que el tratamiento ortodóncico sea realizado haciendo énfasis en los resultados estéticos y funcionales. De esta manera, se trató la maloclusión presente mediante mecánicas que favorecieron la proyección sagital de tejidos duros que, por consecuencia, mejoraron la proyección de tejidos blandos, se llevó a cabo también un control de la dimensión vertical del 


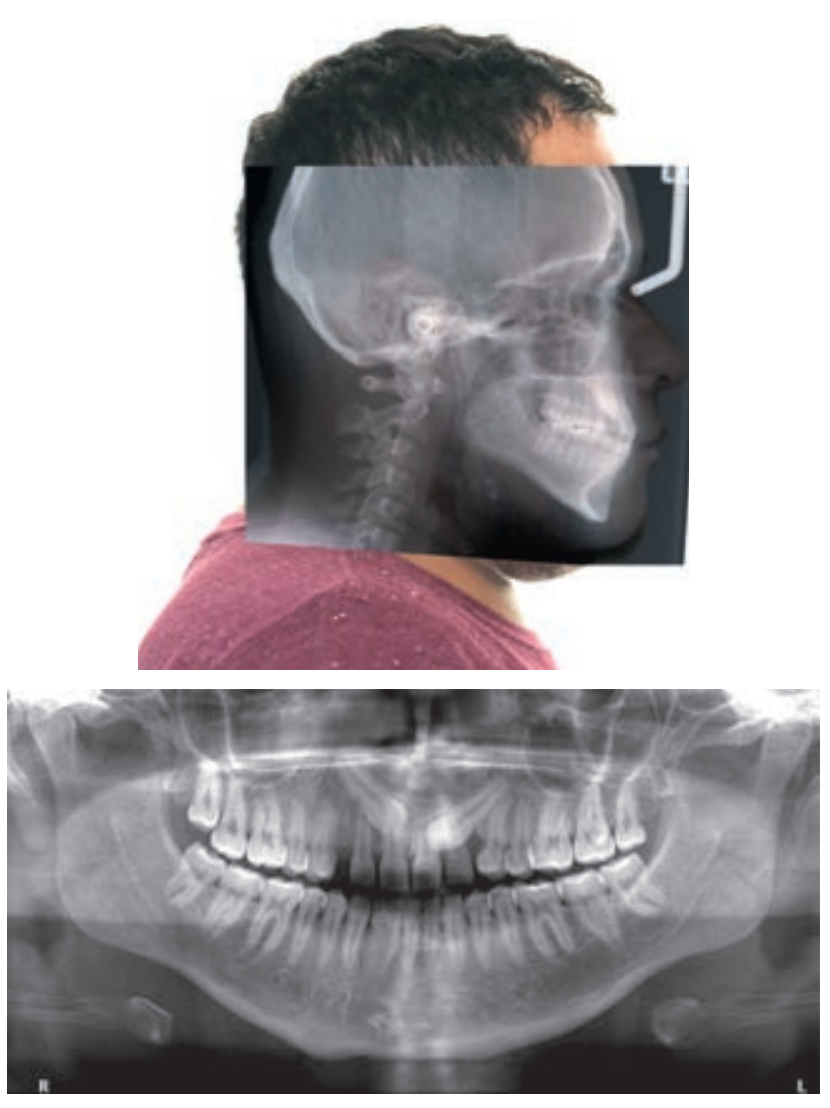

Figura 2: Radiografías iniciales.

tercio inferior con la finalidad de provocar una rotación anterior mandibular para proyectar de manera favorable los tejidos blandos mentonianos.

\section{Progreso del tratamiento}

Para el presente caso, se utilizaron brackets Synthesis de la casa comercial ORMCO con prescripción Roth (slot 0.022) para potenciar el torque y la expresión de los arcos, utilizando una secuencia para la etapa de alineación y nivelación de 0.014 Cuniti $^{\circledR}, 0.016$ Cuniti $^{\circledR}, 0.018$ Cunit $^{\circledR}$ y $0.017 \times 0.025$ Cunit $^{\circledR}$. En esta etapa inicial, se decidió realizar extracciones de los órganos dentales 34 y 44 para reducir la discrepancia óseo-dentaria de la arcada inferior, aumentar el overjet y overbite. Una vez concluida la etapa I, se decidió pasar a la segunda etapa de cierre de espacios y realizar una mecánica de cierre recíproco por medio de cadenas elásticas, en donde al cerrar los espacios de ambas arcadas se llevó una secuencia de arcos de $0.017 \times 0.025 S S$ y 0.019 $\times 0.025$ SS (Figura 5). Al concluir la etapa de cierre de espacios, se procedió a realizar ameloplastias negativas en cúspides palatinas de órganos dentales 14 y 24, los cuales, por cuestión de las anodoncias de los órganos 13 y 23, se decidió que cumplirían con la función de los órganos dentales ausentes mencionados debido a que presentaban buenas características de soporte como una buena longitud radicular y una base ósea adecuada. Además, la posición de los primeros premolares superiores quedo adecuada para establecer una buena relación canina. Una vez realizadas las ameloplastias negativas, se procedió a realizar ameloplastias positivas en los mismos órganos dentales por medio de agregados de resina compuesta para dar una adecuada morfología cuspídea, para que estos órganos puedan realizar adecuadamente la función de guías caninas, lo cual fue uno de los objetivos de tratamiento principales. Al concluir con estas etapas, se procedió a realizar la etapa final en donde se colocaron arcos Braided $0.019 \times 0.025$ para favorecer el asentamiento de mordida. Se dieron indicaciones de realizar ameloplastias positivas con agregados de resina en dientes afectados por la atrición

\section{Retención del caso}

Una vez que se concluyeron las fases activas biomecánicas, se optó por utilizar retenedores circunferenciales para dar estabilidad al caso a largo plazo con indicaciones de uso diurno del retenedor inferior y uso nocturno del retenedor superior. También se hizo uso de láser Quantum
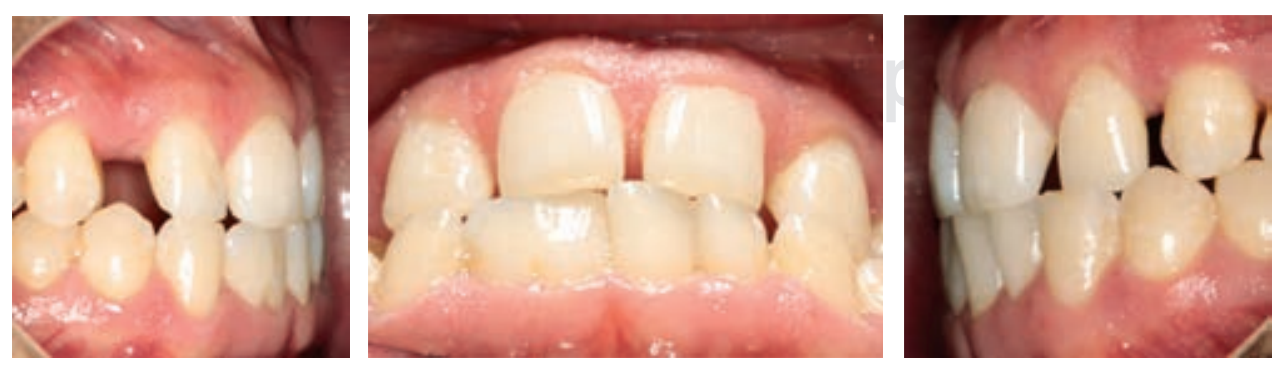

Figura 3:

Overjet y overbite iniciales. 
Figura 4:

Características oclusales de ambas arcadas con punto de contacto determinado.
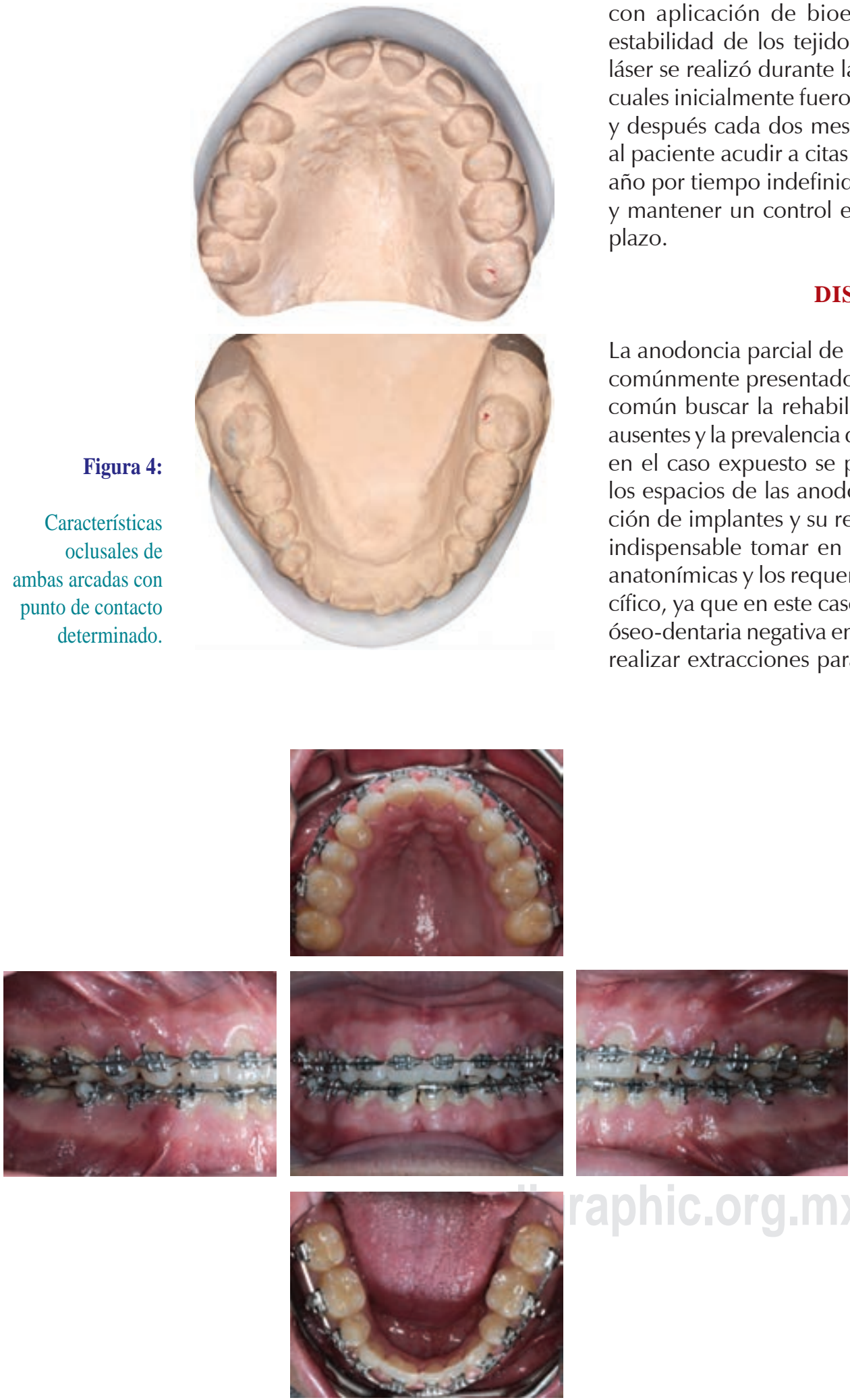

con aplicación de bioestimulación para favorecer la estabilidad de los tejidos de soporte. La aplicación de láser se realizó durante las consultas de seguimiento, las cuales inicialmente fueron indicadas de manera mensual y después cada dos meses durante un año, y se sugirió al paciente acudir a citas de control mínimo dos veces al año por tiempo indefinido para evitar cualquier recidiva y mantener un control estricto de la estabilidad a largo plazo.

\section{DISCUSIÓN}

La anodoncia parcial de caninos superiores es un hecho comúnmente presentado en la práctica ortodóncica. ${ }^{3}$ Es común buscar la rehabilitación de los órganos dentales ausentes y la prevalencia de los tejidos sanos; sin embargo, en el caso expuesto se podría proponer la apertura de los espacios de las anodoncias para la posterior colocación de implantes y su rehabilitación protésica, ${ }^{8}$ pero es indispensable tomar en cuenta todas las características anatonímicas y los requerimientos de cada caso en específico, ya que en este caso, al presentar una discrepancia óseo-dentaria negativa en la arcada inferior, era necesario realizar extracciones para aliviar el apiñamiento y crear
Figura 5:

Arcos de acero previos a finalización. 
una armonía oclusal adecuada que favoreciera la estética y la función. ${ }^{9}$

En los casos que encontramos situaciones de anodoncia parcial, se debe decidir entre mover el premolar a la posición del canino u otras alternativas, como el autotrasplante o la restauración protésica después de realizar un análisis con base en las características y requerimientos que sugiera el caso a tratar. ${ }^{10}$

Se ha demostrado que el éxito de los implantes dentales depende de una adecuada óseo-integración, lo cual se puede ver afectado por distintas circunstancias que se deben considerar, como la falta de higiene bucal, presencia o antecedentes de enfermedad periodontal, ausencia de implicaciones sistemáticas desfavorables para la calidad de las bases óseas, que de por sí en el maxilar superior las características dadas por el hueso trabeculado no siempre son las más favorables. ${ }^{10}$

Es un hecho que las probabilidades de éxito de mantener tejidos propios del organismo en buen estado son más convenientes que recibir tratamientos por medio de aditamentos que intenten cumplir con la función de órganos vitales. ${ }^{11}$

Es importante considerar las desventajas que implica el recibir un tratamiento de rehabilitación protésica, ya que las probabilidades de éxito de mantener tejidos propios del organismo en buen estado son más factibles que recibir tratamientos por medio de aditamentos reemplazan dientes perdidos. ${ }^{10}$

Este tipo de tratamiento será indicado entonces cuando existan discrepancias oseodentarias positivas por anodoncias, en las que las extracciones de más órganos dentales perjudiquen y comprometan la calidad de la oclusión final, así como la estética facial. ${ }^{12}$

Este tratamiento no es recomendado cuando existe una inadecuada longitud radicular, además de un soporte óseo que comprometa la estabilidad a largo plazo de los órganos que serán sometidos a la caracterización morfológica, ya que la carga oclusal que reciben los caninos es distribuida a lo largo de una longitud radicular más amplia en situaciones ideales. Por lo que estos dos parámetros son un factor fundamental a considerar antes de decidir llevar a cabo un tratamiento con estas características. ${ }^{13}$

\section{CONCLUSIONES}

El tratamiento por medio de caninización de premolares es un tratamiento favorable para pacientes que presenten ausencia de caninos permanentes; sin embargo, se deben considerar características específicas, como longitud radicular y estado periodontal, para lograr cumplir con objetivos estéticos y funcionales. Se puede finalizar el caso realizando agregados de resina estéticos para asegurar un buen aspecto y una correcta funcionalidad.

\section{REFERENCIAS}

1. Aguana K, Cohen L. Diagnóstico de caninos retenidos y su importancia en el tratamiento ortodóncico. Revista Latinoamericana de Ortodoncia y Odontopediatria "Ortodoncia. ws edición electrónica junio 2011. Consultada en: www. ortodoncia.ws.

2. Santoyo DC, Calleja Al, García HJ, Díaz RRM. Prevalencia de caninos superiores retenidos en pacientes mexicanos mayores de 14 años de la Facultad de Odontología de la Universidad Tecnológica de México. Rev ADM. 2001; 58 (4): 138-142.

3. Ugalde MFJ, González LR. Prevalencia de retenciones de caninos en pacientes tratados en la clínica de ortodoncia de UNITEC. Rev ADM. 1999; 56 (2): 49-58.

4. Yavuz MS, Aras MH, Büyükkurt MC, Tozoglu S. Impacted mandibular canines. J Contemp Dent Pract. 2007; 8 (7): 78-85.

5. Mutan-Hamdi A, Koray Halicioglu, Muhammed-Selim Yavuz, Murat Caglaroglu. Evaluation of surgical-orthodontic treatments on impacted mandibular canines. Med Oral Patol Oral Cir Bucal. 2011; 16 (7): e925-928.

6. Pacheco C, Dobles AL. Caso clínico: caninos en posición de laterales, resultado estético. Revista Idental. 2014; 99 (6): 427-838.

7. Egido MS, Arnau MC, Juárez El, Jané-Salas E, Marí RA, LópezLópez J. Caninos incluidos, tratamiento odontológico. Revisión de la literatura. Av Odontoestomatol. 2013; 29 (5): 227-238.

8. Valdivia J. Oclusión en prótesis sobre implante. Rev Prot Oseointegr. 2000; 4: 41-59.

9. Morales L, Osorio E. Discrepancia óseo-dentaria severa. Tratamiento ortodóncico sin extracciones. Revista Gaceta Dental [Internet]. 2009 [Citado 2016 febrero 15]: disponible en: http://www.gacetadental. com/2009/03/discrepancia-seo-dentaria-severatratamientoortodncico-sin-extracciones-31297/\# .

10. Domínguez J, Acuña J, Rojas M, Bahamondes J, Matus S. Estudio de asociación entre enfermedades sistémicas y el fracaso de implantes dentales. Rev Clin Periodoncia Implantol Rehabil Oral. 2013; 6 (1): 9-13.

11. Sada ME, Mampaso DC. Consideraciones básicas en implantología oral. Indicaciones y contraindicaciones. Pronóstico, complicaciones y fracasos. Tratamiento. Gaceta Dental. 2000; 106: 38-66.

12. Santiesteban-Ponciano FA. Analysis in calculating Osseo dental discrepancy manually and in I model analysis 2 application. Odontol Sanmarquina. 2016; 19(2): 19-23.

13. Alves Nilton. Morphometric Study of the Dental Roots of Permanent Lower Anterior Teeth in Brazilian Individuals. Int J Morphol. 2015; 33 (1): 210-212. Available from: http:// www.scielo.cl/scielo.php?script $=$ sci_arttext\&pid $=$ S071795022015000100033\&lng=en.

\section{Correspondencia:}

Pablo César Martínez Cardona

E-mail: pcm2312@hotmail.com

Conflicto de intereses: El autor declara no tener ningún conflicto de intereses.

Financiamiento: Ninguno. 